\title{
Working Ni-Mn-Ga Single Crystals in a Magnetic Field Against a Spring Load
}

\author{
P. G. Lindquist ${ }^{1}$ P. Müllner ${ }^{1}$
}

Published online: 21 April 2015

(C) ASM International 2015

\begin{abstract}
This research characterizes ferromagnetic shape memory elements for use as mechanical actuators. A single crystal of Ni-Mn-Ga was pre-strained in compression from 0 to $6 \%$ and then the shape was recovered with a magnetic field perpendicular to the loading direction while working against a pair of springs. The magnetic field was raised from 0 to $0.64 \mathrm{MA} / \mathrm{m}$ and then reduced to zero field. Eight pairs of springs with combined spring constants ranging from 14.3 to $269.4 \mathrm{~N} / \mathrm{mm}$ were used. When the magnetic field was on, the sample expanded against the springs due to magnetic field-induced strain. When the magnetic field was turned off, the springs compressed the sample back to the initial size before the next cycle. During each cycle, force and displacement were measured and the specific work was computed. Specific work increased with the applied magnetic field and the pre-strain, with a maximum of $14 \mathrm{~kJ} / \mathrm{m}^{3}$ at $4.5 \%$ pre-strain and $0.64 \mathrm{MA} / \mathrm{m}$. This value is five times less than the values suggested in the literature which were inferred from stress-strain curves measured under various magnetic fields. The spring prescribes the load-displacement path of the magnetic shape memory element and controls the work output of the actuator.
\end{abstract}

Keywords Ferromagnetic shape memory $\cdot$ Magnet shape memory - Actuator - Actuation work output - Magnetomechanical behavior

\section{P. G. Lindquist \\ paullindquist@boisestate.edu}

1 Department of Materials Science and Engineering, Boise State University, Boise, ID 83725, USA

\section{Introduction}

$\mathrm{Ni}-\mathrm{Mn}-\mathrm{Ga}$ elements have been proposed for use as fast and large-stroke actuators [1-5]. Ni-Mn-Ga single crystals used in spring actuators have actuation stresses from 0.1 to $2 \mathrm{MPa}$ and elongation strains up to $6 \%$ when driven by a magnetic field [3]. These researchers also discussed various actuator designs including push-push actuators, push-pull actuators, and spring-loaded actuators. The push-push actuator makes use of two MSM elements which work against each other to allow bidirectional actuation. This actuator can hold a given position without consuming power. The push-pull actuator has only one MSM element which is pre-loaded by a magnetic field. A variable field drives the MSM element against the bias field. In this case, a constant power is required to hold a position. For the spring-loaded actuator, a spring provides the restoring force. Similar to the push-pull actuator, holding position consumes electrical power.

The shape change is caused by a twin boundary motion [6]. When actuated by a magnetic field, the domain with the crystallography $c$ axis (easy axis of magnetization) parallel to the magnetic field grows. When actuated by a compressive force, the domain with the $c$ axis aligned with the load grows. Thus, in a spring-loaded actuator, the MSM element must be coupled to a magnetic field perpendicular to the spring displacement to cause the sample to expand against the spring force. Cycling under constant stress was shown to recover $2.8 \%$ strain at $1.3 \mathrm{~ms}^{-1}$ as the current to the electromagnet varied 0 and 2A [5]. Recently, higher temperature Ni-MnGa actuator materials were reported with $3 \%$ strain recovery at $350 \mathrm{~K}$ [7]. Smith et al. reported rapid actuation for these materials at room temperature with a twin boundary velocity of $82.5 \mathrm{~ms}^{-1}$ without load [4].

Getting the maximum expansion and contraction from the actuator requires careful design of the magnetic field and the 
spring that the $\mathrm{Ni}-\mathrm{Mn}-\mathrm{Ga}$ element works against. Besides stroke, the work output is also a figure of merit for actuators. Several researchers estimated work output as the product of maximum magnetostress, $\sigma_{\mathrm{M}}$, and maximum twinning strain (also called spontaneous strain $\varepsilon_{0}=1-c / a$, where $c$ and $a$ are tetragonal lattice parameters, [8]). This estimate is an upper limit for an ideal magnetic shape memory alloy where the stress-strain curve is a step function. The work output limitation is also influenced by sample size, as compared to bulk samples with cross sections in the millimeter size. For microactuators with a square cross section of $(200 \mu \mathrm{m} \times$ $200 \mu \mathrm{m})$ and a length of $4 \mathrm{~mm}$ work output increases from 10 to $140 \mathrm{~kJ} / \mathrm{m}^{3}$ as the compressive stress varies from less than 1 to $10 \mathrm{MPa}[9,10]$. Couch and Schlüter et al. [1, 3] measured the stress or strain of the element while it works against the spring. Schlüter et al. reported actuation strains of $3.5 \%(0.7 \mathrm{~mm}$ expansion and contraction for a $20 \mathrm{~mm}$ sample length) for a spring-loaded configuration when a 0-5 A sinusoidal current is applied to an electromagnet. Couch did extensive studies on the magneto-mechanical behavior of $\mathrm{Ni}-\mathrm{Mn}-\mathrm{Ga}$ in order to model the quasi-static mechanical behavior to predict the magnetic shape memory effect and pseudoelasticity [11] and he designed an actuator for trailing edge activation on a helicopter rotor with $2 \mathrm{~mm}$ of travel and $60 \mathrm{~N}$ of force actuation.

This research explores working the active element against springs of varying spring constants, measuring the force and displacement during expansion and contraction of the element while the magnetic field is cycled between 0 and $0.64 \mathrm{MA} / \mathrm{m}$. In this way, we determine directly the work output of the spring-loaded actuator under various loading conditions.

\section{Experiment}

A single crystal with a nominal composition of $\mathrm{Ni}_{51} \mathrm{Mn}_{27} \mathrm{Ga}_{22}$ (numbers indicate atomic percent) was grown with the Bridgman-Stockbarger technique using the crystal growth system developed by Kellis [12]. A portion of the crystal with a $10 \mathrm{M}$ martensite structure and a composition of $\mathrm{Ni}_{51.6}$ $\mathrm{Mn}_{26.3} \mathrm{Ga}_{22.1}$ was aligned such that the $\{100\}$ faces of the crystal matched the surfaces which were cut with a Sodick UZ $300 \mathrm{~L}$ wire electrical discharge machine (EDM). These faces were then polished with 2000 grit $\mathrm{SiC}$ followed by a sequence of diamond pastes to $1 \mu \mathrm{m}$. Afterwards, the crystal was inspected in an optical microscope and cracks from the EDM cutting were found. The crystal was ground again and polished below the surface cracks, yielding a crystal of final size $6.63 \mathrm{~mm} \times 2.77 \mathrm{~mm} \times 3.58 \mathrm{~mm}$ without cracks. A vibrating sample magnetometer (Digital Measurement Systems, Model 10 VSM) with a cryostat for heating and cooling was used to measure the Curie temperature and the austenite to martensite phase transformation temperatures between -50 and $150{ }^{\circ} \mathrm{C}$. An optical microscope (Leica, Model DM6000 $\mathrm{M})$ was used for bright field and differential interference contrast inspection. The composition was determined with electron dispersive spectroscopy (EDS) using a Hitachi 3400 S scanning electron microscope and an Oxford Instruments Energy ${ }^{+}$EDS detector. Crystal alignment and determining the martensite structure was done with a Bruker D8 Discover $\mathrm{X}$-ray diffractometer using a HI-STAR area detector. The magneto-mechanical test apparatus (MMTA) used for measuring work of $\mathrm{Ni}-\mathrm{Mn}-\mathrm{Ga}$ single crystals in a magnetic field was adapted from an earlier apparatus [13] and is shown in Fig. 1. This system consists of (a) a GWM Model 3470 electromagnet with $20 \mathrm{~mm}$ pole pieces set up for magnetic fields between 0 and $0.64 \mathrm{MA} / \mathrm{m}$, (b) a linear variable differential transformer (LVDT) displacement transducer Model MHR-010 from Measurement Specialties with a custom made signal conditioning module scaled to output $\pm 300 \mu \mathrm{m}$ displacement using an AD 698 IC from Analog Devices [14], (c) a Model BM30-10 0-10 mm travel micrometer, $0.01 \mathrm{~mm} / \mathrm{div}$, from Newport Corp. (d) a $44 \mathrm{~N}$ piezoelectric load cell Model 208-C01from PCB Piezotronics Inc., (e) interchangeable pairs of springs with combined spring rates from 14.3 to $269.4 \mathrm{~N} / \mathrm{mm}$ made by Century Springs Inc., (f) Ni-Mn-Ga single crystal test specimen (g) an Ametek Model DLM 600 (60 V and $10 \mathrm{~A})$ programmable power supply. Analog signals for force, displacement, and power supply voltage and current were connected as input to a multiplexed four channel 16 bit analog to digital converter (ADC). The current to the electromagnet was controlled with analog output from a 16 bit digital to analog converter (DAC) which was connected to the power supply remote control. The ADC and DAC inputs and outputs were controlled by a dSpace Model ACE 1104 Power PC embedded controller that was plugged into a personal computer. The embedded controller was programmed from scripts written in MatLab along with a symbolic model for the ADC and DAC converters written in Simulink, an accessory software package from MathWorks. The dSpace system was designed to work with MatLab which had a cross-compiler/linker that downloads the program to the dSpace controller card.

Eight pairs of springs (Century Spring Inc.) with combined spring constants ranging from 14.4 to $269.4 \mathrm{~N} / \mathrm{mm}$ (Table 1) were used as mechanical bias load. The Ni-MnGa crystal was glued with rubber cement (Elmer's Products Inc. 26000 00904) to a brass platen $4.5 \mathrm{~mm}$ in diameter and it was centered between the pole pieces of the magnet. Before testing, the sample was extended to maximum length along the [100] by ramping the magnetic field to $0.64 \mathrm{MA} / \mathrm{m}$ while the sample was between the pole pieces without constraint from the opposite brass platen. This resulted in a single martensite variant with the [001] axis aligned to the magnetic field and the [100] aligned to the axis of mechanical load. 


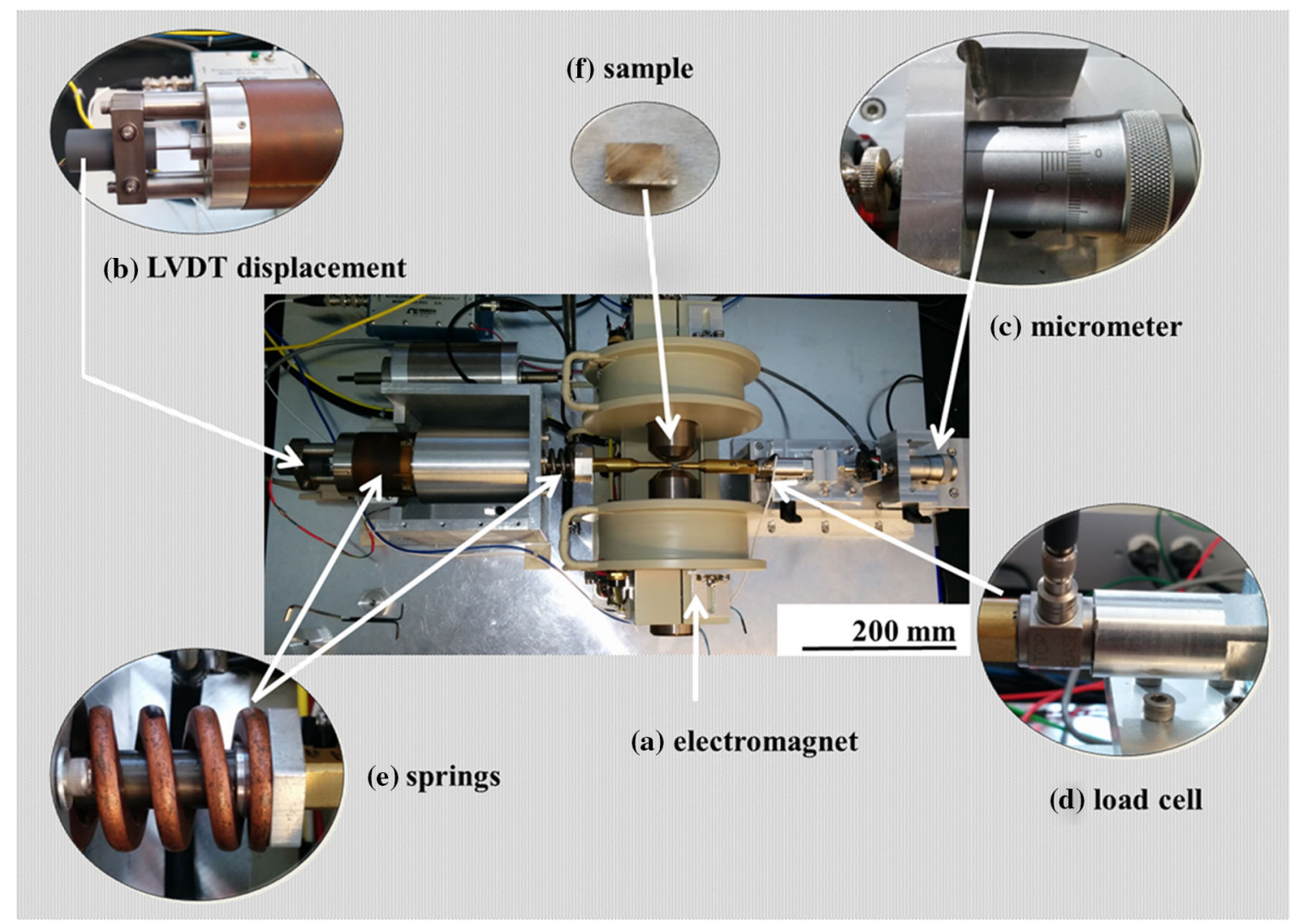

Fig. 1 (Color online) Magneto-mechanical test apparatus (MMTA) used for measurement of the work of Ni-Mn-Ga samples against springs with different spring constants: a two pole electromagnet,

Table 1 Combined spring constants were between 14.3 and $269.4 \pm 0.05 \mathrm{~N} / \mathrm{mm}$

\begin{tabular}{lc}
\hline Spring pair & Combined spring constant $(\mathrm{N} / \mathrm{mm})$ \\
\hline 1 & 14.3 \\
2 & 36.9 \\
3 & 47.0 \\
4 & 71.3 \\
5 & 102.3 \\
6 & 134.4 \\
7 & 135.4 \\
8 & 269.4 \\
\hline
\end{tabular}

The magneto-mechanical test sequence was as follows: (1) compressing the sample $0.05 \mathrm{~mm}$ by displacing the right end of the sample and the load cell to the left by turning the micrometer screw (Fig. 1); (2) incrementally increasing the magnetic field perpendicular to the compression axis from 0 to $0.64 \mathrm{MA} / \mathrm{m}$, in increments of typically $0.04 \mathrm{MA} / \mathrm{m}$; the sample expands to the left with the right end being fixed; (3) reducing the magnetic field by turning off the power supply; (4) repeating steps (2) and (3) totally 10 times; (5) repeating steps (1)-(4) by compressing the sample in step (1) by an additional $0.05 \mathrm{~mm}$ (i.e., by $0.1 \mathrm{~mm}$ ). Experiments were repeated each time with additional b LVDT displacement transducer, c micrometer for compressing the crystal, d piezoelectric load cell, e pair of test springs, f Ni-Mn-Ga single crystal

$0.05 \mathrm{~mm}$ compression until the sample was compressed to a pre-strain of $6 \%$. For step (2, where the duration of each cycle was $1.5 \mathrm{~s}$ ), the magnetic field achieved the desired field strength during the first $0.3 \mathrm{~s}$ after which it was reduced to zero field by shunting the electromagnet to ground through a diode where magnetic field decays due to the resistance $(R)$ and inductance $(L)$ time constant of the system in the next $1.2 \mathrm{~s}$, see Fig. $2 \mathrm{a}$. These times were needed to let the current from the power supply achieve a steady state current thereby a constant magnetic field during ramp up and to let the force and displacement transducers stabilize during ramp up and ramp down of the magnetic field. When the magnetic field was on, the sample expanded and compressed the springs. The magnetic field-induced strain (MFIS) caused the force and displacement of the sample to increase, as measured by the load cell and the displacement transducer (LVDT), Fig. 2b. When the magnetic field was reduced to zero, the compressed springs expanded against the sample and compressed it to the initial length.

For each test cycle, analog inputs for force, displacement, electromagnet voltage, and electromagnet current were sampled from the 16 bit ADC channels every $0.9 \mathrm{~ms}$. The MatLab computer program handled data conversion and scaling, integration, and saved the data for further data analysis. The work done on the sample, $W_{\text {sample, was }}$ 
(a)

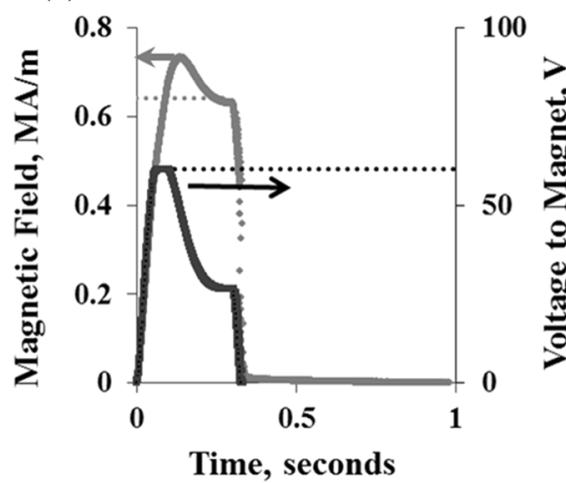

Fig. 2 An example of one cycle of magneto-mechanical testing at a magnetic field of $0.64 \mathrm{MA} / \mathrm{m}$ : a For each cycle, when the magnetic field was on, it took $0.3 \mathrm{~s}$ to get a stable magnetic field of $0.64 \mathrm{MA} / \mathrm{m}$, then the voltage to the electromagnet was set to zero for $1.2 \mathrm{~s}$, from 0.3 to $1.5 \mathrm{~s}$. b As the magnetic field increased with increasing current

computed by integrating the force versus displacement using the trapezoidal rule (1).

$W_{\text {sample }}=\int_{0}^{x_{\max }} F(x) \mathrm{d} x-\int_{x_{\max }}^{0} F(x) \mathrm{d} x$

The force, $F(x)$, was measured with the load cell and the displacement, $x$, was measured with the LVDT at the positions shown in Fig. 1 . To calculate specific work, $W_{\text {sample }}$ was divided by the sample volume.

In addition to magneto-mechanical cycling against springs, the magnetostress and MFIS were measured under static magnetic fields. To obtain compressive stress-strain curves of the samples under magnetic fields of $0,0.16,0.28$, and $0.4 \mathrm{MA} / \mathrm{m}$, a soft spring $(14.3 \mathrm{~N} / \mathrm{mm})$ was used and the sample was slowly compressed with the stage micrometer while the magnetic field was held constant. Knowing the sample compression and the spring deflection, stress and strain were then computed. Spring deflection was measured by the LVDT displacement transducer. The stress was computed by multiplying the spring rate times the spring deflection divided by the cross section of the sample. The strain was calculated by measuring the spring deflection and the movement of the stage micrometer to obtain $\Delta l$ and dividing by the fully expanded sample length, $l_{0}(6.629 \mathrm{~mm})$. Obtaining a stressstrain curve at $0.64 \mathrm{MA} / \mathrm{m}$ was not possible because above 2 MPa the spring deflected beyond the LVDT detection range.

\section{Results}

From XRD the structure of the Ni-Mn-Ga crystal was $10 \mathrm{M}$ and EDS analysis indicated a composition of $\mathrm{Ni}_{51.6}$ $\mathrm{Mn}_{26.3} \mathrm{Ga}_{22.1}$. The phase transformation temperatures found from low magnetic field testing in the VSM as the

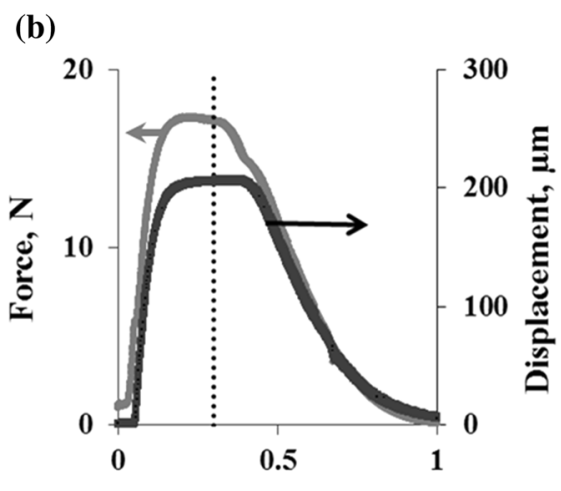

Time, seconds

to the electromagnet, the force on the sample increased to $17 \mathrm{~N}$ and the sample displaced the springs $205 \mu \mathrm{m}$. When the magnetic field was ramping down, the springs compressed the crystal to the initial length

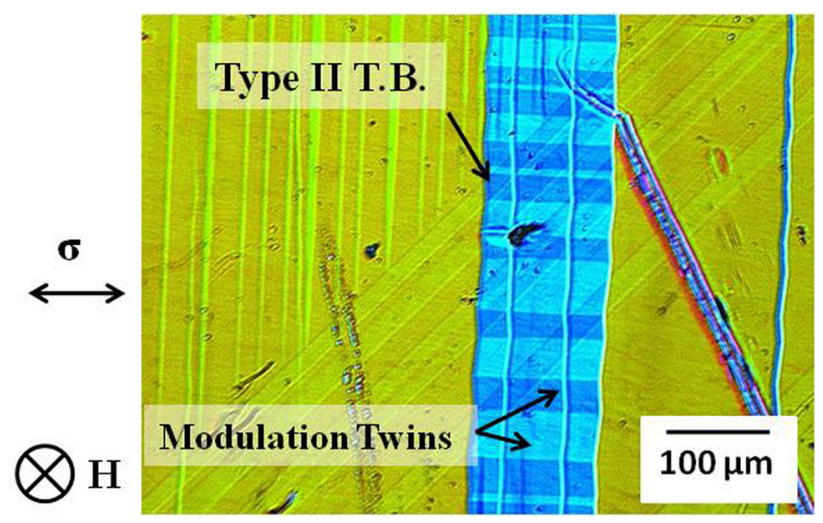

Fig. 3 (Color online) Differential interference contrast optical micrograph showing Type II twin boundaries along with modulation twins after the sample was compressed $3.6 \%$. The blue Type II twins grew as the compression on the sample increased. The diagonal relief pattern indicates historic twins [15] which are unrelated to the currently active twins. The direction of the magnetic field $\mathrm{H}$ and the compression/expansion axis $\sigma$ is shown

temperature of the sample was heated and cooled between -50 to $150{ }^{\circ} \mathrm{C}$ were: $M_{\mathrm{s}}$ of $45^{\circ} \mathrm{C}, M_{\mathrm{f}}$ of $34.1^{\circ} \mathrm{C}, A_{\mathrm{s}}$ of $41.4{ }^{\circ} \mathrm{C}$ and $A_{\mathrm{f}}$ of $49.8^{\circ} \mathrm{C}$. The Curie temperature was $89{ }^{\circ} \mathrm{C}$. The single crystal was observed with the optical microscope at various times during the testing and at differing levels of compressive strain. Straight twin boundaries indicative of Type I twinning were observed. Type II twin boundaries were also present at low and high number of magneto-mechanical cycles. In Fig. 3, a serrated Type II twin boundary along with internal modulation twins were observed in the sample after it was compressed $3.6 \%$. The thickness of the Type II twin boundaries increased as the sample was compressed. The micrograph was taken after thousands of magneto-mechanical cycles, so there are 
Fig. 4 (Color online) Data for spring pair $5(102 \mathrm{~N} / \mathrm{mm})$ : a Specific work as the sample was compressed from 0 to $6 \%$ strain while the magnetic field varied from 0 to $0.64 \mathrm{MA} / \mathrm{m}$. b Maximum specific work was achieved at $4.5 \%$ strain and $0.64 \mathrm{MA} / \mathrm{m}$ magnetic field (a)

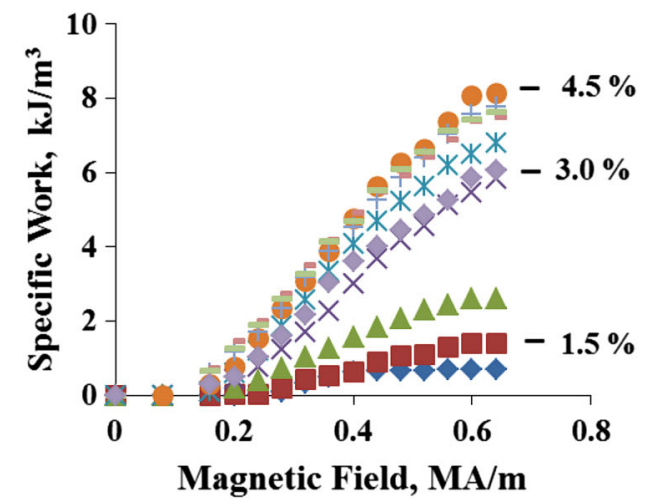

(b)

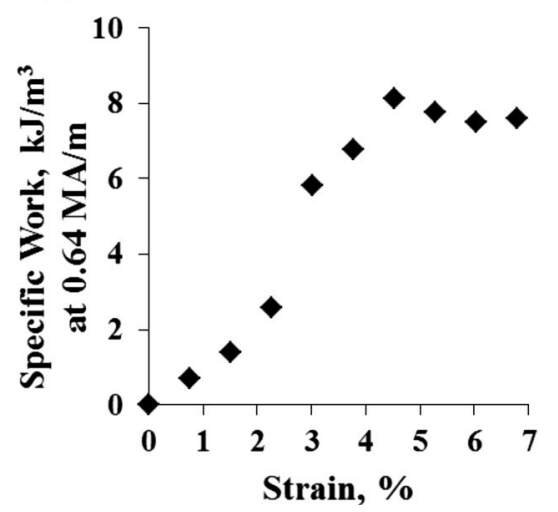

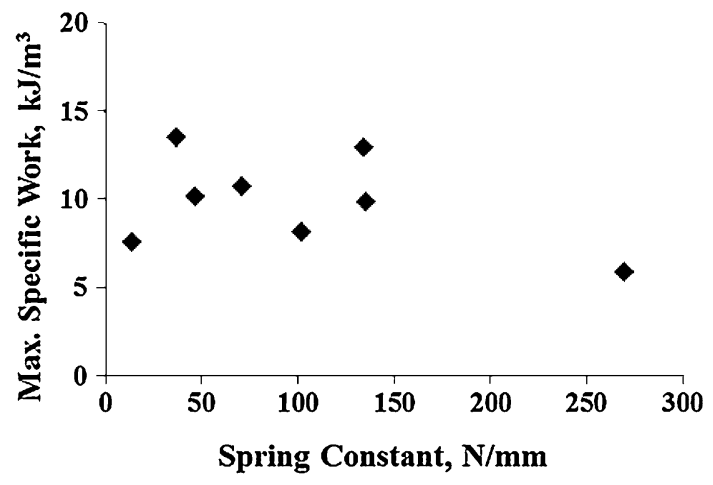

Fig. 5 The maximum specific work for samples cycled in a magnetic field of $0.64 \mathrm{MA} / \mathrm{m}$ at optimum pre-strain

historic twins and features in the micrograph that were unrelated to the active twins [15]. Specific work for one series of tests using a spring pair with a spring constant of $102 \mathrm{~N} / \mathrm{mm}$ is shown in Fig. 4a. Here the sample pre-strain varied from 0 to $6 \%$ strain and the magnetic field varied from 0 to $0.64 \mathrm{MA} / \mathrm{m}$. The maximum specific work was achieved at $4.5 \%$ strain and $0.64 \mathrm{MA} / \mathrm{m}$ magnetic field, Fig. 4b. A threshold magnetic field of $0.16 \mathrm{MA} / \mathrm{m}$ was required to initiate deformation leading to detectable work output. For this set of springs, the maximum specific work of $8.1 \mathrm{~kJ} / \mathrm{m}^{3}$ was reached when the sample was prestrained at $4.5 \%$.

For all springs, specific work increased with magnetic field and reached a maximum at the maximum field of $0.64 \mathrm{MA} / \mathrm{m}$. Specific work also depended on how much the sample was compressed prior to magnetic field cycling. Obtaining the maximum specific work required compressing the sample 4-5\% prior to magnetic field cycling. The maximum specific work density ranged from 8 to $14 \mathrm{~kJ} / \mathrm{m}^{3}$ without a systematic dependence on spring constant as shown in Fig. 5. Maximum MFIS and maximum magnetostress are shown in Fig. 6a, b. At low spring constants, the sample extended almost $5 \%$ with the maximum stress reaching $0.8 \mathrm{MPa}$ for each magnetic cycle. At the highest spring constant of $269.4 \mathrm{~N} / \mathrm{mm}$, the sample did not expand beyond $0.4 \%$ reaching a load of 2.3 MPa. Below the threshold field, the magnetostress increased linearly with the magnetic field at $\frac{\partial \sigma}{\partial H}=5.8 \mathrm{~N} / \mathrm{Am}$.

The compressive stress-strain curves at magnetic fields of $0,0.16,0.28$, and $0.4 \mathrm{MA} / \mathrm{m}$ are shown in Fig. 7. For these plots, stress and strain were positive for compression. At zero magnetic field, as the sample was compressed the stress increased without any strain until the stress reached the twinning stress, then the sample deformed with a slight increase in stress, the twinning stress varied from 0.23 to $0.27 \mathrm{MPa}$ from 0.5 to $6 \%$ strain. With the magnetic field constant and above $0.4 \mathrm{MA} / \mathrm{m}$, the stress required to deform the sample increased with magnetic field up to $2 \mathrm{MPa}$. The dashed arrows indicate the path taken when the sample was compressed to $4.5 \%$ pre-strain prior to starting magnetic field cycling. Superimposed on the stress-strain curve are the stress-strain curves obtained by magnetic field cycling against spring set 5 with a spring constant of $102 \mathrm{~N} / \mathrm{mm}$ at a magnetic field of 0.16 ( $\boldsymbol{\Delta}$ purple), 0.28 ( $\boldsymbol{\square}$ olive green), 0.40 ( red), and 0.64 (aqua) MA/m. When the magnetic field was cycled up and down, the stress-strain response against the springs fell exactly between the curve for zero magnetic field and the magnetostress-strain curve obtained at the respective magnetic field. When the magnetic field reached the set field, the stress on the sample increased until the twinning stress plus the opposing spring stress exceeded, then the sample expanded following a linear stress-strain path until the stress reached the stress-strain curve for the applied magnetic field. The slope of the linear path was determined by the spring constant, Table 1 . When the magnetic field was reduced to zero, the stress diminished and then the springs compressed the sample back along a linear stress-strain path with the same slope as for the loading ramp. For the first cycle, there was a $0.26 \mathrm{MPa}$ jump in the stress level between the zero magnetic field stressstrain curve and the magneto-mechanical loops. 
Fig. 6 a Maximum MFIS recovered for each cycle was up to $5 \%$ for low spring constants and less than $1 \%$ for high spring constants. b The magnetostress reached a maximum of $2.2 \mathrm{MPa}$ for high spring constants (a)

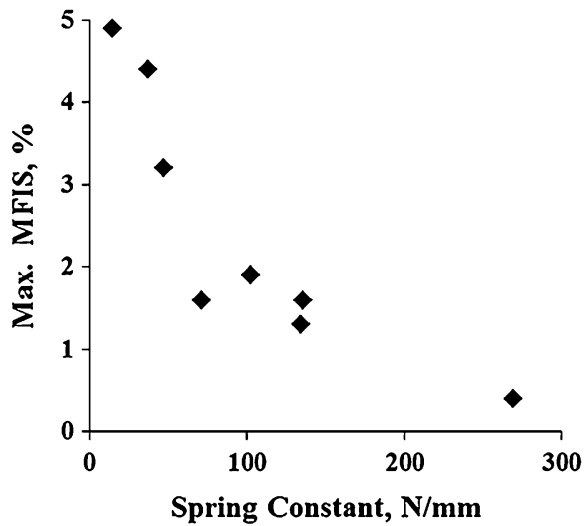

(b)

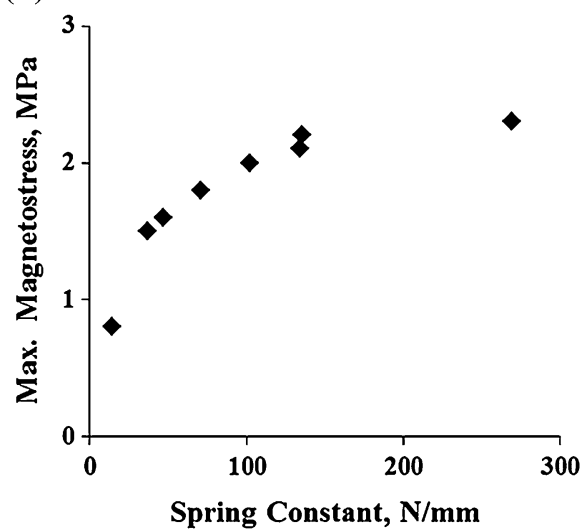

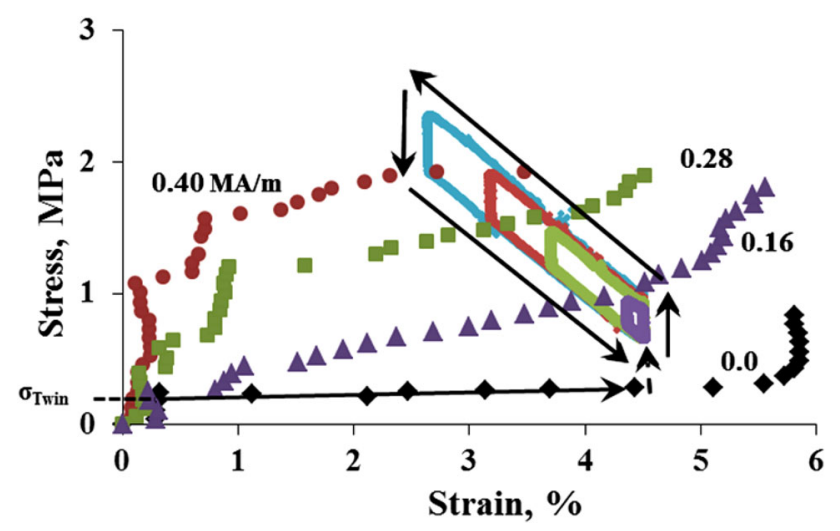

Fig. 7 (Color online) Stress-strain curves as the sample was compressed from 0 to $6 \%$ strain while holding the magnetic field constant at $0 \mathrm{MA} / \mathrm{m}$ (black diamond black), 0.16 (black up-pointing triangle purple), 0.28 (black square olive green), and 0.40 (black circle red). For these plots, stress and strain were positive for compression and there was not a $0.64 \mathrm{MA} / \mathrm{m}$ stress-strain curve plotted. The arrows indicate the path taken when the sample was compressed to $4.5 \%$ strain prior to starting magnetic field cycling. Superimposed on the stress-strain curve are the stress-strain curves obtained by cycling (nine cycles per test) against spring pair 5 $(102 \mathrm{~N} / \mathrm{mm})$ at magnetic fields of 0.16 (black up-pointing triangle purple), 0.28 (black square olive green), 0.40 (black circle red), and 0.64 (black line aqua) $\mathrm{MA} / \mathrm{m}$
For spring pairs with combined spring constants from 14.3 to $269.4 \mathrm{~N} / \mathrm{mm}$, the stress-strain of the crystal expanding and contracting against the springs were plotted in Fig. 8a. The path direction of each magneto-mechanical cycle was the same as shown with arrows in the Fig. 7. The MFIS start stress to expand against the spring pairs increased with increasing spring constant as shown in Fig. 8b. MFIS start stress was independent of pre-strain and varied from 0.91 to $1.1 \mathrm{MPa}$ from 0.56 to $5.27 \%$ pre-strain, Fig. 9.

\section{Discussion}

Knowing how work output, force, and displacement change with spring constant is useful for developing design guidelines for spring-loaded actuators. This magneto-mechanical test system differs from a screw-driven mechanical tester by (1) the piezoelectric load cell being stiffer than a strain-gage-based load cell, and (2) containing interchangeable springs that the sample works against when it expands due to the applied magnetic field. These modifications allow measurement of the work output of the sample with different spring constants in a set-up similar to
Fig. 8 a Stress-strain curves obtained from cycling (nine cycles per test) against spring pairs with combined spring constants that ranged from 14.3 to $269.4 \mathrm{~N} / \mathrm{mm}$. b The MFIS start stress varied from 0.56 to 1.9 $\mathrm{MPa}$ when the spring constant changed (a)

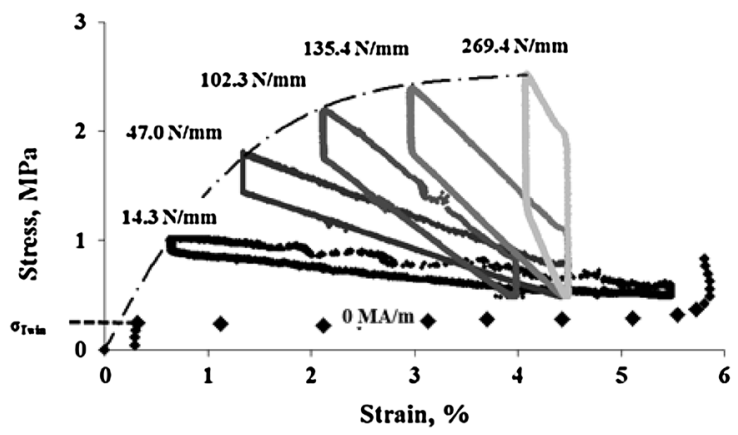

(b)

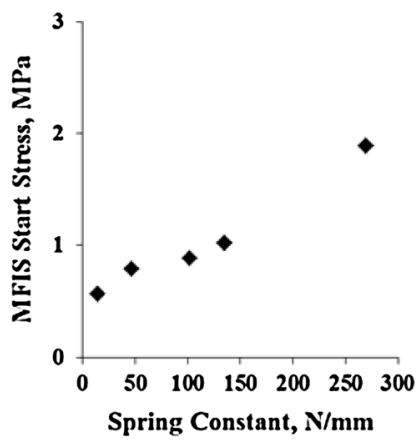


how the elements are used in a spring-loaded actuator [3]. For this study, the demagnetization factor not corrected for because the change in magnetization as a function of strain was not measured [16].

From our results three findings are apparent. (1) It is possible to predict the useful load a spring-loaded actuator is capable of lifting and the distance the actuator will move. (2) The work output of a spring-loaded actuator is less than that obtained when computing the area under the stress-strain curve. (3) High spring constants require higher magnetostress before expansion and low spring constants require lower magnetostress. Using the relationship similar to one proposed by Sozinov et al. [17] and an earlier relation reported by Heczko and Straka [16, 18], the actuator begins to move when the magnetostress, $\sigma_{\mathrm{mag}}$, exceeds the sum of the spring stress, $\sigma_{\text {spring, }}$, the actuator load, $\sigma_{\text {act.load }}$, the frictional stress, $\sigma_{\text {frict }}$, and twice the twinning stress $\sigma_{\mathrm{TW}}$ as shown in Eq. (2).

For loading:

$\sigma_{\text {spring }}+\sigma_{\text {act.load }}+\sigma_{\text {frict }}+2 \sigma_{\mathrm{TW}}<\sigma_{\text {mag }}$

With increasing magnetic field, $\sigma_{\mathrm{mag}}$ increases. The sample will expand if $\sigma_{\text {mag }}$ exceeds the sum of the stresses resulting from the spring load, the actuator load (which is zero here), and the friction of the apparatus and twice the twinning stress. The twinning stress appears with the factor two because the magnetic field must compensate the stress which was required to compress the sample by moving the right side of the sample with the micrometer screw (i.e., step 1 described in the experimental procedure) which equals the twinning stress and the magnetic field must move twin boundaries in the opposite direction to elongate the sample. Thus, the magnetic field must overcome the twinning stress twice. The friction stress increases with the transverse force which is in first approximation proportional to the spring load. Thus, without spring, magnetic field-induced straining should start at $2 \sigma_{\mathrm{TW}}$ (Fig. 8b) shows the stress at the onset of MFIS as a function of the spring constant. At zero spring constant, the onset stress is $0.5 \mathrm{MPa}$ which is twice the twinning stress of $0.25 \mathrm{MPa}$ (Fig. 8a). The friction force increases with overall load and, thus, the stress hysteresis of the loops shown in Fig. 8a increases with increasing spring constant.

Measurement of the maximum work output while expanding and contracting against the spring requires prestraining the sample prior to magneto-mechanical cycling. An alternate method proposed by Schlüter et al. [3] was to pre-stress the sample. For a single crystal with a twinning stress of $0.5 \mathrm{MPa}$ they used a pre-load of 0.6 MPa. With this pre-load and the springs chosen they achieved an actuation strain of approximately $2 \%(20 \mathrm{~mm}$ element length and a $0.3 \mathrm{~mm}$ length change). The results of work output depended on the twinning stress. To characterize the work output independent of twinning stress, pre-straining is

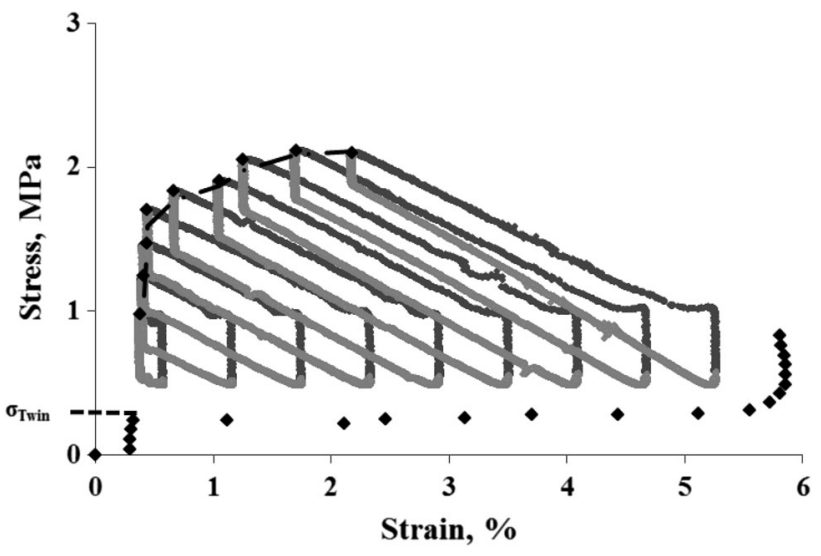

Fig. 9 Stress-strain curves obtained from cycling (nine cycles per test) against spring pair $2(36.9 \mathrm{~N} / \mathrm{mm})$ while varying the pre-strain before magneto-cycling. The MFIS start stress ranged from 0.91 to 1.1 $\mathrm{MPa}$ for all pre-strains tested

preferred because slope of the stress-strain curve is relatively flat from 0 to $6 \%$ strain. For pre-stress, small changes in stress cause larger changes in strain. When strain is fixed the stress equalizes to that point on the stress-strain curve.

In this research, the specific work is determined by measuring force and displacement of $\mathrm{Ni}-\mathrm{Mn}-\mathrm{Ga}$ against a spring. The resulting work output values are $2-5$ times lower than values reported by other researchers. However, those reports list the product of magnetostress and maximum strain as work output. This value is in fact not the actual work output but it is an upper bound for the attainable work output. Researchers [8, 19] summarized their measurements and those made by other researchers [20-23]. In Fig. 10, our results are plotted together with the results reported by the other researchers. The iso-energy lines shown represent the product $W_{\text {ideal }}$ of maximum magnetostress and spontaneous strain $W_{\text {ideal }}=\sigma_{\mathrm{M}} \varepsilon_{0}$. Our results range from 9.2 to $66 \mathrm{~kJ} / \mathrm{m}^{3}$ which were slightly lower than the results from Heczko and Murray. The major difference is that the spring-loaded testing only achieved $5.3 \%$ strain recovery compared to $6 \%$ in their results, so there is a lower work output in our results. Karaca found $60 \mathrm{~kJ} / \mathrm{m}^{3}$ but those measurements were made at $-95{ }^{\circ} \mathrm{C}$ while we and other authors performed experiments at room temperature. Other researchers [24] performed experiments where they compressed the sample in a mechanical test bench. They then applied a constant magnetic field orthogonal to the loading direction and slowly moved the cross head to allow the sample to expand in the magnetic field while recording the force on the sample. When the sample was fully expanded, they removed the magnetic field and compressed the sample without magnetic field. The area between the expansion and compression force-displacement curves was about $110 \mathrm{~kJ} / \mathrm{m}^{3}$. Similar to the results reported in Fig. 10, this method provides an upper bound of attainable 


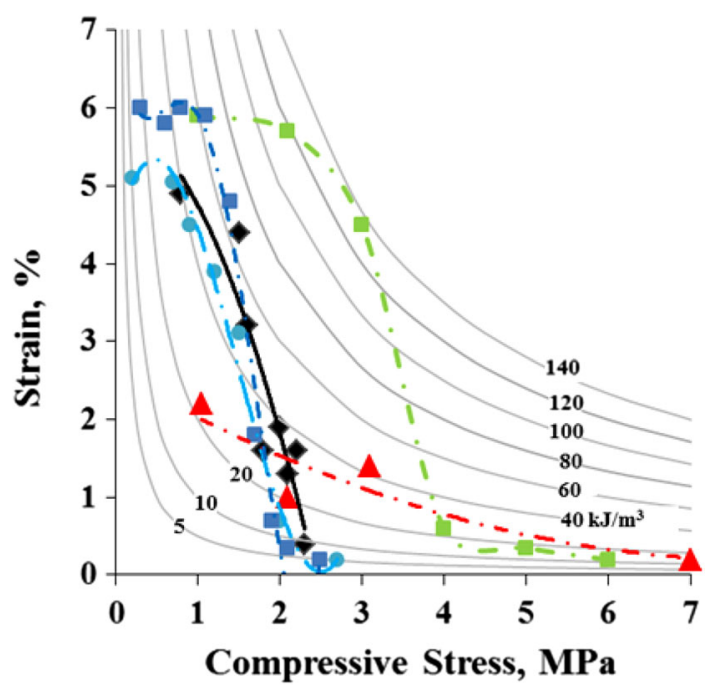

Fig. 10 (Color online) Compressive stress, strain, and specific work as reported by others and analyzing our data in the same way. Current results (black diamond solid line, $25{ }^{\circ} \mathrm{C}$ ) were similar to [20] (black circle dashed line, $25^{\circ} \mathrm{C}$ ) and [22] (black square dashed line, $25^{\circ} \mathrm{C}$ ). The results of [23] (black up-pointing triangle dashed line, $-18{ }^{\circ} \mathrm{C}$ ) and [19] (black square dashed line, $-95^{\circ} \mathrm{C}$ ) were also plotted

work output. The loading/unloading path does not correspond to a purely magnetically driven actuator. Thus, these results do not compare to the results obtained with our experiments which are run under purely magnetic actuation.

Determining the relationship between specific work and spring rate is limited by the experimental set-up and the active twinning system. For our experimental set-up, it is difficult to find springs that have precise spring rates and are manufactured to close dimensional tolerances. The variations in spring quality affect the side loading on the linear bearing. Eliminating the friction from the side loading is important because the forces measured are often below $5 \mathrm{~N}$ and the friction forces add significantly to the forces measured. Additional experiments are planned to remove the bearing assembly from the apparatus or to use low friction air bearings. The second issue is the nature of active twins. Based on the research of Straka et al. [25], the twinning stress of Type I twins is temperature dependent and ranges typically from 1 to $2 \mathrm{MPa}$ and Type II twins have a twinning stress that is independent of temperature and typically less than $0.25 \mathrm{MPa}$. From the stress-strain curves shown in Fig. 8 (diamond symbols), a twinning stress of 0.23 to $0.27 \mathrm{MPa}$ is indicative of Type II twinning. In this case, the specific work measured ranges from 8 to $14 \mathrm{~kJ} / \mathrm{m}^{3}$. If the crystal sits for a couple of weeks, sometimes the specific work measured is in the range of $25 \mathrm{~kJ} / \mathrm{m}^{3}$ which supports activation of Type I twins instead of the expected Type II twins. The higher twinning stress of Type I twins increases the area of the force-displacement curve resulting in a higher specific work.

\section{Conclusions}

The conclusions of this study are

- The active twinning system in a spring-loaded actuator can be Type I or Type II twinning.

- In this study, specific work did not vary systematically with the spring constant.

- Low spring constant actuators lead to high expansion and contraction of the actuator.

- High spring constant actuators support higher stresses but less actuation length.

- The effective work output of a purely magnetically driven actuator is two to five times smaller than the upper bounds obtained from the product of maximum magnetostress and twinning strain or by following an actuation path which includes varying the mechanical load without magnetic field.

Acknowledgments We thank Eric Rhoads for growing the Ni-MnGa single crystal, Brittni Blessie for preparing the sample, Andrew Morrison for optical microscopy, Sam Barker, Eric Rhoads, and Theodore Lawrence for design and fabrication of the magneto-mechanical test system, and Aaron Smith for valuable discussions. We acknowledge financial support from the US Department of Energy's Office of Basic Energy Sciences under Contract DEFG-0207ER46396 for this study and from the National Science Foundation Major Research Instrumentation program through DMR-0619795 (XRD).

Conflict of interest The authors declare that they have no conflict of interest.

\section{References}

1. Couch RN (2006) Development of magnetic shape memory alloy for a swashplateless rotor. $\mathrm{PhD}$ thesis, University of Maryland, $\mathrm{MD}$

2. Marioni M, O'Handley RC, Allen SM (2003) Pulsed magnetic field-induced actutation of Ni-Mn-Ga single crystals. Appl Phys Lett 83:3966-3968

3. Schlüter K, Holz B, Raatz A (2012) Principle design of actuators driven by magnetic shape memory alloys. Adv Eng Mater 14:682-686

4. Smith AR, Tellinen J, Ullakko K (2014) Rapid actuation and response of Ni-Mn-Ga to magnetic-field-induced stress. Acta Mater 80:373-379

5. Suorsa K, Pagounis E, Ullakko K (2004) Magnetic shape memory actuator performance. J Magn Magn Mater 272:2029-2030

6. Ullakko K (1996) Magnetically controlled shape memory alloys: a new class of actuator materials. J Mater Eng Perform 5:405-409

7. Pagounis E, Laptev A, Jungwirth J, Laufenberg M, Fonin M (2014) Magnetomechanical properties of a high-temperature NiMn-Ga magnetic shape memory actuator material. Scripta Mater $88: 17-20$

8. Karaca H, Karaman I, Basaran B, Chumlyakov Y, Maier H (2006) Magnetic field and stress induced martensite reorientation in NiMnGa ferromagnetic shape memory alloy single crystals. Acta Mater 54:233-245 
9. Ganor Y, Shilo D, Messier J, Shield TW, James RD (2007) Testing system for ferromagnetic shape memory microactuators. Rev Sci Instrum 78:073907

10. Ganor Y, Shilo D, Shield TW, James RD (2008) Breaching the work output limitation of ferromagnetic shape memory alloys. Appl Phys Lett 93:122509

11. Couch RN, Chopra I (2007) A quasi-static model for NiMnGa magnetic shape memory alloy. Smart Mater Struct 16:S11-S21

12. Kellis D, Smith A, Ullakko K, Müllner P (2012) Oriented single crystals of Ni-Mn-Ga with very low switching field. J Cryst Growth 359:64-68

13. Lindquist PG, Lindquist G, Rothenbuhler A, Müllner PM (2013) Dynamic measurement of the mechanical behavior of ferromagnetic shape memory alloys for power generation. Paper presented at the international conference of shape memory alloys, Boise, ID, 7 June 2013

14. AD698 Universal LVDT Signal Conditioning (1995). Analog Devices, One Technology Way, PO Box 9106, Norwood, MA

15. Reinhold M, Watson CS, Knowlton WB, Müllner P (2010) Transformation twinning of Ni-Mn-Ga characterized with temperature-controlled atomic force microscopy. J Appl Phys 107:113501

16. Straka L, Heczko O (2005) Reversible $6 \%$ strain of Ni-Ni-Ga martensite using opposing external stress in static and variable magnetic fields. J Magn Magn Mater 290-291:829-831

17. Sozinov A, Lanska N, Soroka A, Zou W (2013) $12 \%$ magnetic field-induced strain in Ni-Mn-Ga-based non-modulated martensite. Appl Phys Lett 102:021902
18. Heczko O, Straka L (2003) Temperature dependence and temperature limits of magnetic shape memory effect. J Appl Phys 94:7139-7143

19. Kiefer B, Karaca HE, Lagoudas DC, Karaman I (2006) Characterization and modeling of the magnetic field-induce strain and work output in Ni2MnGa magnetic shape memory alloys. J Magn Magn Mater 312:164-175

20. Heczko O, Sozinov A, Ullakko K (2000) Giant field-induced reversible strain in magnetic shape memory NiMnGa alloy. IEEE Trans Magn 36:3266-3268

21. James RD, Tickle R, Wuttig M (1999) Large field-induced strains in ferromagnetic shape memory alloys. Mater Sci Eng A 273-275:320-325

22. Murray SJ, Marioni M, Allen SM, O'Handley RC, Lograsso TA (2000) $6 \%$ magnetic-field-induced strain by twin-boundary motion in ferromagnetic Ni-Mn-Ga. Appl Phys Lett 77:886-888

23. Tickle R, James RD, Shield T, Wuttig M, Kokorin VV (1999) Ferromagnetic shape memory in the NiMnGa system. IEEE Trans Magn 35:4301-4310

24. Schiepp T, Detkov V, Pagounis E, Laufenberg M (2013) Failure mechanisms and high-cycle fatigue of MSM actuators. Paper presented at the international conference of shape memory alloys, Boise, ID, 3 June 2013

25. Straka L, Soroka A, Seiner H, Hanninen H, Sozinov A (2012) Temperature dependence of twinning stress of Type I and Type II twins in $10 \mathrm{M}$ modulated $\mathrm{Ni}-\mathrm{Mn}-\mathrm{Ga}$ martensite. Scripta Mater 67:25-28 